\title{
Design of the Power Amplifier for Magnetic Bearings Based on the Three-level PWM Modulation
}

\author{
Xiaodan Ren ${ }^{1,}$, Qiang Yin ${ }^{1,}$, Yue Yu, c, Hao Pang ${ }^{1}$, Zecheng Xiong ${ }^{1}$ and \\ Qiliang Zhao' \\ ${ }^{1}$ XJ Power CO.,LTD.,XJ Group Corporation, Xuchang 461000,Henan, China \\ arenxiaodandy@126.com, byin-1-qiang@163.com, ${ }^{c}$ yuyuedydy@126.com
}

\begin{abstract}
Keywords: Electronics, Three-level, PWM modulation, Power amplifier, Magnetic bearings.
Abstract. As it is scant for the linear power amplifier and the two-level switching power amplifier, the principle is analyzed for the three-level power amplifier. It is proposed for the power amplifier for magnetic bearings based on the three-level PWM modulation technique. The circuit is designed for the implementation of the function. The two triangular carriers are formed by the triangular wave superimposing the positive and negative voltage bias, and then, the two drives are formed by comparing the current error signal and the two triangular carriers based on the voltage comparator. The experiment results show that the method can achieve the three-level work, reduce the output current ripple, and have good characteristics. The conclusions have some guiding significance to the design of the three-level power amplifier for magnetic bearings.
\end{abstract}

\section{Introduction}

Power amplifier is a very important part for magnetic bearings in the control system. It can provide enough coil current to generate electromagnetic force which can realize stable suspension and affect the bearing capacity and the dynamic performance of the system. The linear power amplifier is mostly adopted in the magnetic bearings system in the early. Because of the shortcomings of the linear power amplifier, such as, the low efficiency, the relatively large volume and the small suitable region, the switching power amplifier is almost used now, with the high efficiency and good dynamic characteristic. The two-level modulation technique is adopted in the switching power amplifier for magnetic bearings early. In view of the disadvantages, such as, the large ripple current and the limited dynamic characteristics, the three-level modulation technique is studied in recent[1-5].

Power amplifier has three main technical index. They are the current tracking characteristics, the dynamic characteristics and the output current ripple value. Aiming at the shortages of the linear power amplifier and the two-level switching power amplifier, the principle of the three-level power amplifier is analyzed, the power amplifier for magnetic bearings based on the three-level PWM modulation is proposed, and the circuit is designed for the implementation of the function. The two triangular carriers are formed by the triangular wave superimposing the positive and negative voltage bias, and then, the two drives are formed by comparing the current error signal and the two triangular carriers based on the voltage comparator. The control circuit composed of the analog circuit components has the advantage of simple structure. It can realize low ripple current in the coil and has good dynamic characteristics.

\section{The three-level power amplifier}

The structure diagram of three-level power amplifier is shown in Fig.1. Q1 and Q2 are the power MOSFET. D1 and D2 are the power fast recovery diode. The bus voltage is supported and the power tube peak is absorbed with the electrolytic capacitor E1 and non-inductance capacitor $\mathrm{C} 1$. $\mathrm{Rm}$ is the coil resistance for magnetic bearings. Lm is the coil inductance for magnetic bearings. The coil current is sampled by the current transformer series in the circuit. 


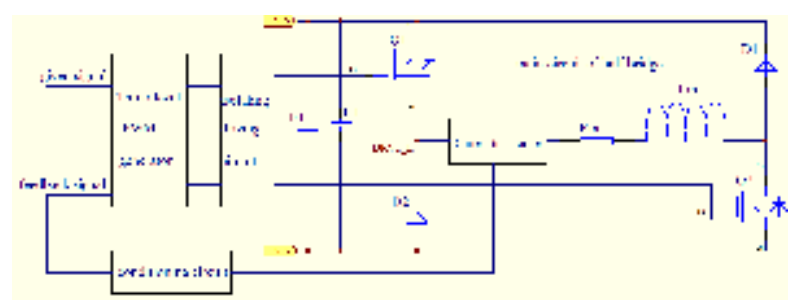

Fig. 1 the structure diagram of three-level power amplifier

The three-level power amplifier has three kinds of working states. They are the charge state, the discharge state and the current reflowing state. By switching among three kinds of working states, the coil current can track the given current in order to achieve the purposes of power amplification. The equation of current ripple of the three-level modulation is shown in formula (1)[4]. Where, $\tau=\mathrm{Lm} / \mathrm{Rm}$ is the time constant of the coil. Therefore, the ripple current is independent of DC bus voltage.

$I_{V-t r i}=\sqrt{\frac{2 e^{-(t / \tau)}+\frac{t}{\tau} e^{-(t / \tau)}-2+\frac{t}{\tau}}{2\left[e^{-(t / \tau)}-1\right]}}$

The schematic of charge state is shown in Fig.2. Q1 and Q2 are open at the same time, while D1 and D2 are close at the same time. The coil current increases in this state. The coil voltage is the supply voltage by neglecting power tube voltage drop. The circuit of charge state is Q1, current transformer, Rm, Lm and Q2. The schematic of current reflowing state is shown in Fig.3. Q1 is open, while Q2 is close. D2 is open, while D1 is close. The coil current flows along the same direction. The circuit of charge state is D2, current transformer, Rm, Lm and Q2. The schematic of discharge state is shown in Fig.4. Q1 and Q2 are close at the same time, while D1 and D2 are open at the same time. The coil current decreases in this state. The coil voltage is the reverse supply voltage by neglecting the diode conduction voltage drop. The circuit of discharge state is D2, current transformer, Rm, Lm and D1. [3][4]

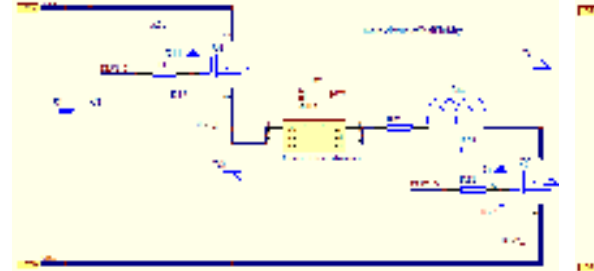

Fig. 2 the charge state

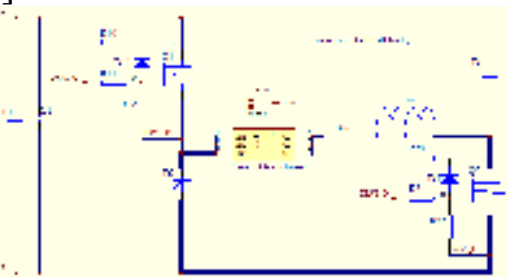

Fig. 3 the current reflowing state

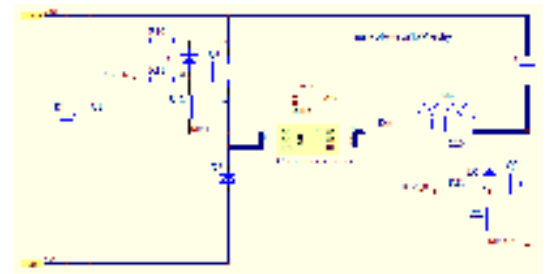

Fig. 4 the discharge state

\section{The design of three-level PWM power amplifier}

The three-level power amplifier is designed based on the principle analysis and the structure diagram. The PWM generation circuit includes error comparator, current PI regulator, triangular wave generator, voltage bias circuit and voltage comparator. The current feedback signal is obtained by the signal conditioning circuit dealing with the coil current, and then the current error signal is get with the current PI regulator through comparing the given signal and the feedback signal. Subsequently, the two triangular carriers signal are obtained by the triangular wave superimposing the voltage bias. The voltage bias is implemented with the voltage bias circuit. The two driving signals are obtained by voltage comparator through comparing the two triangular carriers signal with the current error signal. Finally, The power tubes are open and close by two conditioning driving signal through the driving circuit and isolation circuit. So, the purpose of controlling load current is achieved. The principle block diagram of three-level PWM modulation is shown in Fig.5, and the principle formation diagram of three-level PWM modulation is shown in Fig.6. 


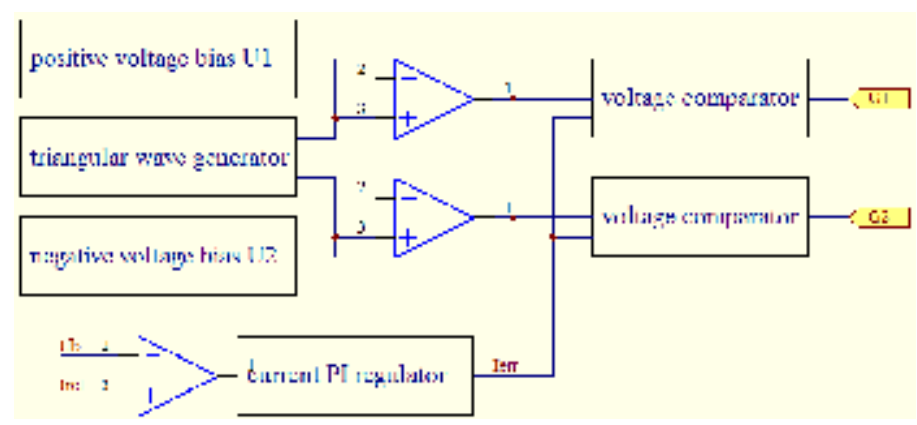

Fig.5 the principle block diagram

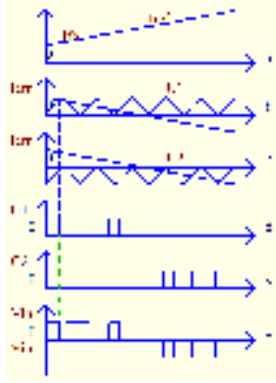

Fig.6 the principle formation diagram

The circuit diagram of the triangular carrier in the three-level PWM modulation is shown in Fig.7a.The triangular wave is into the non-inverting input terminal of amplifier through the amplifier tracking and the resistance R2. The supply $\mathrm{VCC}$ is into the non-inverting input terminal of amplifier through the resistance R3. VCC can be a positive voltage or negative voltage. The voltage is the positive bias, when VCC is a positive voltage. The voltage is the negative bias, when VCC is a negative voltage. And then, the two triangular carriers are obtained through the amplifier tracking.

The circuit diagram of the current error signal in the three-level PWM modulation is shown in Fig. $7 b$. The IF signal is obtained by the signal conditioning circuit dealing with the current feedback signal. The IGA signal is obtained by the filter. The current error signal EO is obtained by the current PI regulator and the high noise rejection ratio, high precision operational amplifier. The PI parameter is determined by the resistor R34, the capacitor C34 and the capacitor C33.

The circuit diagram of the driving circuit in the three-level PWM modulation is shown in Fig.7c. The triangular carrier is into the inverting input terminal of voltage comparator. The current error signal is into the non-inverting input terminal of voltage comparator. The pulse voltage square wave signal is obtained by voltage comparator output. The GX pulse voltage is obtained by the gate circuit through the pulse voltage square wave signal and the protection signal OFF.

DRV1_G and DRV2_G are obtained by the driving circuit and isolating circuit for pulse voltage driving square wave signal of G1 and G2. Then, Q1 and Q2 are directly driven. The purpose of controlling load current is achieved by power tubes on and off respectively.

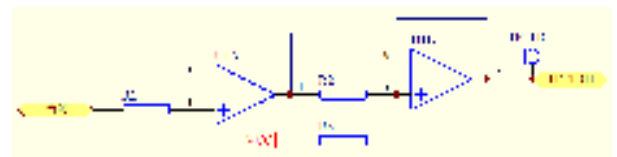

(a)

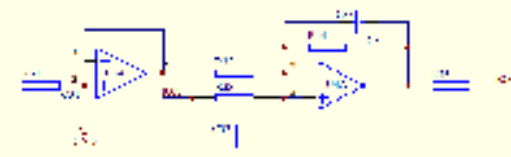

(b)

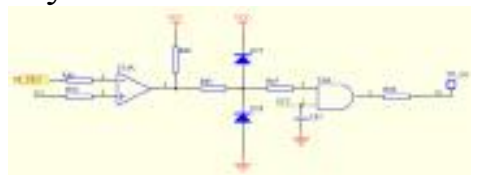

(c)

Fig.7a) the triangular carrier circuit b) the current error signal circuit c) the driving circuit generator

\section{Experiment}

According to the operation principle and the circuit implementation, the power amplifier for magnetic bearings based on the three-level PWM modulation was designed and manufactured. The DC bus voltage is $200 \mathrm{VDC}$. The maximum output current is $20 \mathrm{~A}$. The coil inductance is $10 \mathrm{mH}$. The coil resistance is less than $1 \mathrm{ohm}$.

The ratio between the input voltage and the output current is $3 \mathrm{~A} / \mathrm{V}$. The conversion of LAH-50NP current sensor is 2000:1. The CH1 is the given current channel. The $\mathrm{CH} 2$ is the current feedback channel. The $\mathrm{CH} 3$ is the upper tube driving signal channel. The $\mathrm{CH} 4$ is the under tube driving signal channel. The driving waveform of the current rising process is shown in Fig.8a. The driving waveform of the current falling process is shown in Fig.8b. The output current is shown in Fig.8c as $2 \mathrm{~V}$ of each lattice and $10 \mathrm{~ms}$ of each lattice in the situation of a given DC bias $0.7 \mathrm{~V}$ and a sine wave AC component, in which the amplitude is $0.3 \mathrm{~V}$ and the frequency is $50 \mathrm{~Hz}$. The output current is shown in Fig.8d as $2 \mathrm{~V}$ of each lattice and $10 \mathrm{~ms}$ of each lattice in the situation of a given $\mathrm{DC}$ bias $4 \mathrm{~V}$ and a sine wave $\mathrm{AC}$ component, in which the amplitude is $2.1 \mathrm{~V}$ and the frequency is $50 \mathrm{~Hz}$. The output current is shown in Fig.8e as $2 \mathrm{~V}$ of each lattice and $40 \mathrm{~ms}$ of each lattice in the situation of a given DC bias $4.5 \mathrm{~V}$ and a sine wave AC component, in which the amplitude is $0.5 \mathrm{~V}$ and the frequency is $10 \mathrm{~Hz}$. The output current is shown in Fig. $8 \mathrm{f}$ as $2 \mathrm{~V}$ of each lattice and $1 \mathrm{~ms}$ of each 
lattice in the situation of a given $\mathrm{DC}$ bias $4.5 \mathrm{~V}$ and a sine wave $\mathrm{AC}$ component, in which the amplitude is $0.5 \mathrm{~V}$ and the frequency is $500 \mathrm{~Hz}$.

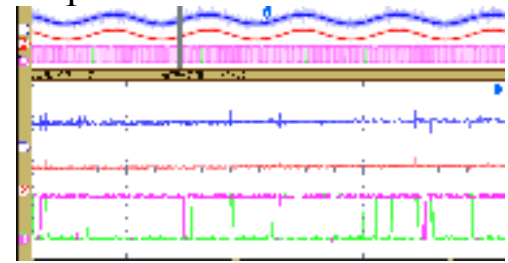

(a)

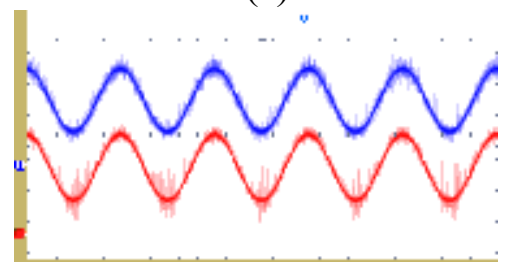

(d)

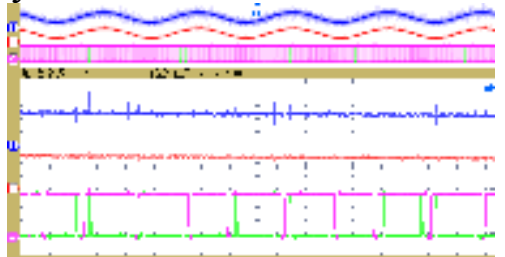

(b)

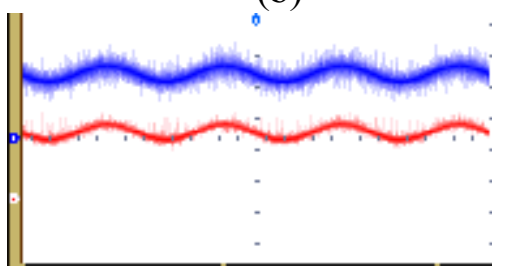

(e)

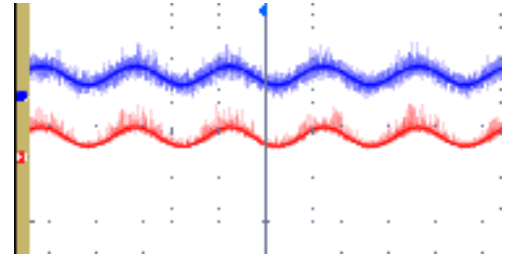

(c)

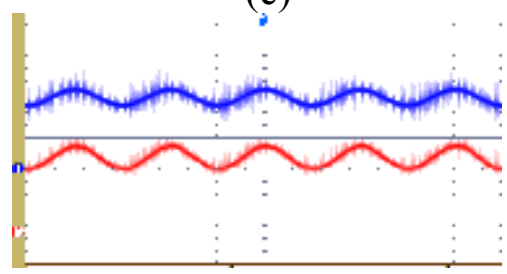

(f)

Fig. 8a) the waveform of the current rising b) the waveform of the current falling c) the waveform of $0.7 \mathrm{VDC}+50 \mathrm{~Hz} / 0.3 \mathrm{VAC} \mathrm{d}$ ) the waveform of $4.0 \mathrm{VDC}+50 \mathrm{~Hz} / 2.1 \mathrm{VAC}$ e) the waveform of 4.5 $\mathrm{VDC}+10 \mathrm{~Hz} / 0.5 \mathrm{VAC}$ f) the waveform of $4.5 \mathrm{VDC}+500 \mathrm{~Hz} / 0.5 \mathrm{VAC}$

It can be seen that the power amplifier for magnetic bearings can achieve three-level working states from Fig.8a to Fig.8b. It can be seen that the output current ripple is small, current signal distortion is small, the output current can quickly track the input signal changes and has good steady state characteristics from Fig.8c to Fig.8d. It can be seen that the output current ripple is small, the output current can rapidly track the input signal changes and has good dynamic state characteristics from Fig.8e to Fig.8f.

\section{Summary}

The principle of the three-level power amplifier is analyzed. The equation of current ripple of the three-level modulation is given. The power amplifier for magnetic bearings based on the three-level PWM modulation is proposed. The circuit is designed for the implementation of the function. The prototype was designed and manufactured. It can be seen from the experiment that three state working mode is achieved, the output current ripple is reduced, and then has good dynamic characteristics, tracking characteristics and steady state characteristics. The conclusions have some guiding significance to the design of the three-level power amplifier for magnetic bearings.

\section{References}

[1] WANG Jun, XU Long-xiang. System Modeling and Control for Switching Power Amplifier of Magnetic Bearing[J].China Mechanical Engineering, 2010,21(4):477-481

[2] ZHAI Xiao-fei, LIU De-zhi, OUYANG Bin, etc. Current ripple reduction with symmetrical PWM for H-half bridge switching power amplifier[J]. Electric Power Automation Equipment, 2011,31(2):58-61

[3] ZANG Xiao-min, WANG Xiao-lin, QIU Zhi-jian, etc. Research on Current Mode Tri-state Modulation Technology in Switching Power Amplifier for Magnetic Bearings[J]. Proceedings of the CSEE, 2004,24(9):167-172

[4] Zhang Jing. Power amplifier for magnetic bearings[D].Switzerland: Swiss Federal Institute of Technology, 1995Stackelberg H. The Theory of the Market Economy. Oxford: Oxford University Press, 1952.

[5] ZHOU Dan, ZHU Chang-sheng. Modulations of Current-mode Switching Power Amplifier for Active Magnetic Bearings[J]. Journal of Mechanical Engineering, 2010,46(20):1-8 